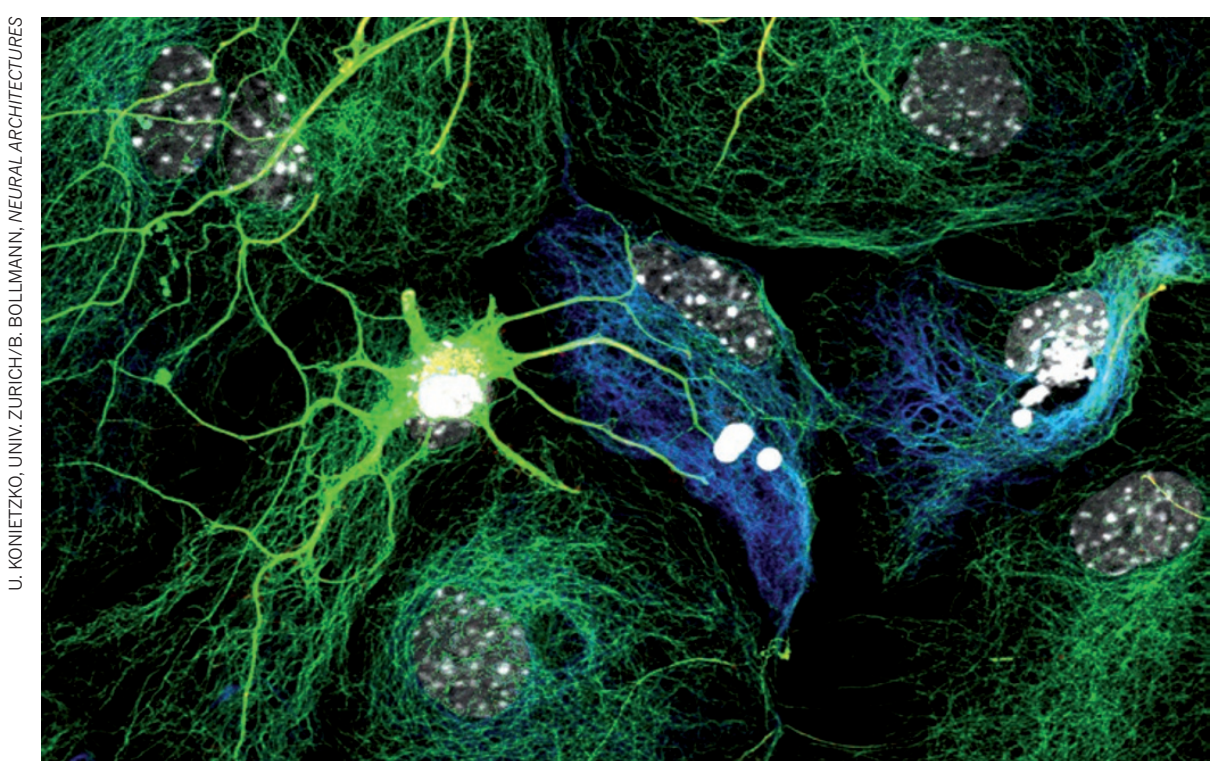

A microscope image of brain glia and neurons reveals the tangle of connections in this mental 'jungle'.

\title{
NEUROSCIENCE
}

\section{The connected self}

\section{Christof Koch tours the idea that the essence of the mind lies in the links between neurons.}

\section{$\mathrm{W}$}

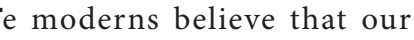
momentary, subjective experience is intimately linked to events in the brain. One set of neurons fires, and we perceive an apple's colour, while a different population of cells gives rise to its taste. Yet the self is also stable: turn the brain off, as happens during heart surgery when the body is cooled to frigid temperatures, and on recovery, the patient's character, personality, habits and long-term memories remain intact. It is these stable aspects of the self, rather than the ebb and flow of our thoughts and percepts, that physicist-turned-neuroscientist Sebastian Seung seeks to explain in Connectome.

Seung argues intelligently and powerfully that the self lies in the totality of the brain's wiring - the eponymous 'connectome', the word used by neuroscientists to denote all the fibre bundles (the white matter) of the human brain. These insulated nerve axons have a total length of around 150,000 kilometres. Seung hails a new science, 'connectomics', as the key to understanding the brain and its pathologies.

This view is grounded in a older doctrine known as connectionism, which postulates that neurons are simple devices and that their connections determine their functions. Cataloguing the links among neurons therefore charts the mind.

\section{DNATURE.COM}

For Nature's special issue on autism: go.nature.com/ypogf8
The heart of Connectome deals with how nervous systems can be reconstructed using electron microscopy. To do this, neural tissue is cut into slices 40-50 nanometres thick, and then imaged to a resolution of a few nanometres. Imaging 1 cubic millimetre of cortex generates 1 petabyte of data, or about a billion photo images from a typical

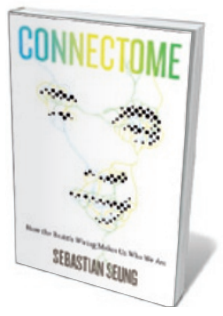

Connectome: How the Brain's Wiring Makes Us Who We Are

SEBASTIAN SEUNG Houghton Mifflin Harcourt: 2012. 384 pp. $\$ 27$ digital camera.

Yet taking the pictures is not the hardest part. Far more daunting is labelling and tracing each neuronal process as it wends its way through the stack of images. Done manually, this would take a trained technician a million working years for each cubic millimetre of brain; luckily, computer vision and machine-learning algorithms speed things up. Last year, two teams reported in Nature partial reconstructions of small volumes in the mouse retina and cortex.

Seung's companion through the neural labyrinth is Hollywood star Jennifer Aniston. In 2005, my group, collaborating with neurosurgeon Itzhak Fried, discovered individual neurons that respond selectively to the image or the name of familiar or famous individuals, such as your grandmother or Aniston. Seung reasons that such neurons must receive input from many less specific cells that respond to eye and hair colour and so on. These in turn connect to neurons responding to even more elementary stimuli, such as vertical lines. The essence of a person's concept of a celebrity lies, then, not with the individual neuron, but in its wiring. And this, in Seung's view, is why reconstructing connections is the key to understanding the mind.

Not even Aniston's agent would think that people are born with neurons that recognize her. Rather, the neural networks that feed into these highly selective cells have wired themselves up to respond in this way. Seung discusses what we know about such plasticity, describing how mammalian nervous systems rewire themselves to adapt to the environment, balance the competing demands of plasticity and stability (so new memories don't displace old ones) and compensate for damage by rerouting information.

Treating the connectome as the be-all and end-all of brain function has its problems. Seung, for example, rebrands autism and schizophrenia as 'connectopathies' diseases in which the brain's wiring goes awry. Yet plenty of other things are wrong in brains with these disorders besides their connectivity.

Faults in synaptic transmission and in processes inside neurons and the glial cells that support them have all been implicated in mental illness and brain disease. Neurons are intricate devices with elaborate input structures that show complex, time-dependent and nonlinear processing. They have various characteristic, and often tortuous, morphologies. Connectionism treats all this as irrelevant. Even though we have known the connectome of the nematode worm for 25 years, we are far from reading its mind. We don't yet understand how its nerve cells work.

The book is well illustrated and sourced, with an ending that is both engaging and idiosyncratic. The last two chapters are predicated on the idea that "you are your connectome", as Seung quips. One deals with technologies to freeze and pickle dead brains long term, in the hope of one day reviving their minds. The final chapter looks at the possibility of simulating the entire human brain on a future supercomputer, allowing you to upload yourself and live on as a software construct. This is the dream of the singularity that Ray Kurzweil and other technologists are hoping will render them immortal: the rapture for nerds.

Christof Koch is chief scientific officer at the Allen Institute for Brain Science, Seattle, Washington, USA, and a professor of biology and engineering at the California Institute of Technology, Pasadena, California, USA. e-mail:koch@klab.caltech.edu 